\title{
Is M235T polymorphism of the angiotensinogen gene involved in the development of endometriosis?
}

\author{
Liliana J. Kowalczyńska' ${ }^{1}$, Tomasz Ferenc ${ }^{1}$, Michał Wojciechowski ${ }^{2}$, Anna Mordalska ${ }^{1}$, \\ Krzysztof Pogoda ${ }^{2}$, Andrzej Malinowski ${ }^{2}$ \\ ${ }^{1}$ Department of Biology and Medical Genetics, Medical University of Lodz, Poland \\ ${ }^{2}$ Department of Operative Gynecology, Endoscopic and Gynecologic Oncology, \\ Polish Mother's Memorial Hospital — Research Institute, Lodz, Poland
}

\begin{abstract}
Objectives: The aim of the study was to analyze the M235T polymorphism of the angiotensinogen (AGT) gene in women with endometriosis and to identify correlations between identified genotypes and the disease progression, its stage and clinical course as well as to evaluate the prognostic value of the investigated polymorphism in patients with endometriosis treated for infertility.

Material and methods: The study group consisted of 241 women with minimal to severe stage of endometriosis, the control group (without endometriosis) - 127. The molecular analysis was performed by PCR-RFLP technique.

Results: The analysis of the frequency of genotypes and alleles of M235T polymorphism showed no significant differences between the study and the control groups and between the severity grades of the disease $(p>0.05)$. No such differences were reported in the case of different localizations of the disease lesions, either. Evaluation of the correlations related to pain accompanying endometriosis did not demonstrate association with any genotypes of the analyzed AGT gene polymorphism. Comparison of the results obtained in the group in which infertility treatment was successful $(n=54)$ and in those who failed to conceive $(n=73)$ did not show the correlation between the investigated polymorphism and the effect of infertility treatment.

Conclusions: M235T polymorphism of the AGT gene seems unrelated to the development or the clinical course of endometriosis. No prognostic value has been found of the investigated polymorphism in predicting the effects of infertility treatment in women with endometriosis.
\end{abstract}

Key words: endometriosis, RAS system, angiotensinogen, gene polymorphism, infertility

Ginekologia Polska 2017; 88, 11: 585-590

\section{INTRODUCTION}

Endometriosis is a chronic disease characterized by the presence of endometrial implants outside the uterus, most commonly in the peritoneum, pelvis and ovaries where cysts are often formed. It affects about $10 \%$ of women in their reproductive years [1, 2]. Clinically it is often manifested by chronic pain (menstrual, perimenstrual, dyspareunia, pelvic pain) and fertility problems. It is estimated that $30-50 \%$ of women with endometriosis have difficulty getting pregnant $[3,4]$. The amount of pain a woman experiences is not necessarily related to the severity of the disease. Some women with severe endometriosis may experience no pain, while others with a milder form of the disease may have severe pain [1].

Endometriosis was first described more than three centuries ago [5]. Despite intense research over many years, the etiopathogenesis of this disease remains unclear. The most common hypothesis for the development of endometriosis is Sampson's theory, which assumes that during menstruation some of the exfoliated mucosal cells flow backwards through the fallopian tubes into the abdomen, where they take root and grow $[3,6]$. Other frequently mentioned and already classical theories explaining the development of endometriosis are the theory of metaplasia and the theory of 
Table 1. Characterization of the study group (women with endometriosis)

\begin{tabular}{|c|c|c|c|}
\hline Endometriosis stage & Number of patients & Mean age & Range of age \\
\hline I & 61 & $31.8 \pm 4.8$ & $21-45$ \\
\hline II & 61 & $33.5 \pm 5.8$ & $24-52$ \\
\hline III & 91 & $32.5 \pm 6.7$ & $17-50$ \\
\hline Total & 70 & $31.2 \pm 5.4$ & $20-46$ \\
\hline
\end{tabular}

induction. The involvement of immune and environmental factors is also indicated [2, 7]. Furthermore, risk factors for disease development and progression include, among others, Caucasian race, age 25 to 35 years, positive family history of endometriosis, early menarche (below 11 years of age) and short menstrual cycles (less than 25 days) with heavy bleeding $[8,9]$. The genetic background is also important. Numerous studies have proven that endometriosis can be classified as hereditary. It occurs more frequently in women whose first-degree relatives also have endometriosis [2]. These assumptions are also confirmed in the study on monozygotic twins [10]. Furthermore, the importance of angiogenesis in the development and survival of endometriosis in the peritoneal cavity has been emphasized in recent years [11, 12].

In the hormonal cascade of the renin-angiotensin system (RAS), the angiotensinogen is released from the liver and cleaved in the peripheral blood by renin to form the decapeptide angiotensin I. Angiotensin I is then converted, by removal of two end-terminal amino acids, to form an octapeptide angiotensin II (Ang II) by the angiotensin-converting enzyme (ACE). Ang II is considered to be the most active component of RAS. Angiotensin Il exerts its actions on different tissues and organs by interacting with two major subtypes of $G$ protein-coupled receptors - angiotensin II type 1 and type 2 receptors (AT1R and AT2R) [13]. One of the effects of the activation of these receptors is the stimulation of the synthesis of vascular endothelial growth factors (VEGF) which directly induce the formation of new blood vessels [14].

\section{OBJECTIVES}

The aim of this study was to analyze the M235T polymorphism of the angiotensinogen gene in women with endometriosis and to determine the correlation between the identified genotypes and the stage of the disease as well as its clinical course. An attempt was also made to estimate the prognostic value of the analyzed polymorphism in patients with endometriosis treated for infertility.

\section{MATERIAL AND METHODS}

\section{Subjects}

The study group included 283 women, mean age 32 years (range 17-52), treated at the Department of Ope- rative Gynecology, Endoscopy and Gynecologic Oncology, Polish Mother's Memorial Hospital-Research Institute in Lodz, diagnosed upon laparoscopic examination with different stages (I-IV) of endometriosis according to the revised American Fertility Society (rAFS) classification system which is characterized in Table 1 [15].

The control group consisted of 127 women (mean age $39.6 \pm 10.4$, range 20-66) without endometriosis treated at the same Department.

The study protocol was approved by the Bioethical Committee of the Medical University of Lodz (RNN/421/12/KB).

\section{Molecular analysis}

Peripheral venous blood samples were collected from all subjects into tubes containing $\mathrm{Na}_{2}$ EDTA. Genomic DNA was isolated from white blood cells, using the Blood Mini kit (DNA-Gdańsk, Poland) and stored at $-20^{\circ} \mathrm{C}$.

The M235T polymorphism of the angiotensinogen gene was typed by polymerase chain reaction-restriction fragment length polymorphism (PCR-RFLP) analysis. A 165-bp DNA fragment containing the polymorphic site was amplified using forward primer 5 '-CCG TTT GTG CAG GGC CTG GCT CTC T-3' and reverse primer 5'-GCC AGG GTG CTG TCC ACA CTG ACT CCC-3'. The PCR reaction was performed in a $50 \mu \mathrm{l}$ volume containing $100 \mathrm{ng}$ of each primer, $0.1 \mathrm{mM}$ of dNTPs, $1 \times$ PCR buffer, $1.5 \mathrm{U}$ of Taq polymerase (Fermentas) and $50 \mathrm{ng}$ of genomic DNA. The amplification conditions were as follows: $94^{\circ} \mathrm{C}$ for $7 \mathrm{~min}, 35$ cycles of $94^{\circ} \mathrm{C}$ for $45 \mathrm{~s}, 65^{\circ} \mathrm{C}$ for $40 \mathrm{sec}$ and $72^{\circ} \mathrm{C}$ for $40 \mathrm{sec}$, followed by final extension at $72^{\circ} \mathrm{C}$ for 7 min (Genius, Techne). A $10 \mu$ l aliquot of the PCR product mixtures was digested with $1 \mathrm{U}$ of FastDigest Boxl (Thermo Scientific) restriction enzyme for 20 minutes. Digestion products were visualized through ethidium bromide staining after electrophoresis in $2 \%$ agarose gels. The electrophoresis revealed a $165 \mathrm{bp}$ fragment for the homozygotes $(M M), 165$ and $141 \mathrm{bp}$ fragments for heterozygotes (MT) and $141 \mathrm{bp}$ fragment for homozygotes (TT). The $24 \mathrm{bp}$ fragment was not seen after digestion.

\section{Statistical analysis}

The statistical analysis was performed using Statistica $10.0 \mathrm{pl}$. The $\mathrm{chi}^{2}$ test was applied in statistical calculations 
for the analysis of variables. $\mathrm{P}<0.05$ was considered statistically significant.

\section{RESULTS}

To accomplish the research goal related to the evaluation of the correlation between the M235T polymorphism of the angiotensinogen gene and the development and progression of endometriosis there were examined 283 patients with a diagnosed disease and 127 control women without endometriosis. The frequency of the analyzed genotypes $(M M, M T, T T)$ did not demonstrate statistically significant differences between the control and the study group $(p=0.15)$ (Table 2). Similarly, no differences were found between the four stages of the disease $(p=0.68)$ (Table 2$)$. Furthermore, the frequency of $A G T$ gene $M$ and $T$ alleles were determined in the analyzed systems but statistically significant differences were not apparent, either (Table 2).

Due to the lack of statistical differences in the analyzed systems, two additional subgroups were distinguished in the study group: $A(n=122)$ including patients with stage I and II and group $B(n=161)$ with stage III and IV of endometriosis acc. to the rAFS classification system [15]. In this case, in group A the distribution of $\mathrm{M} 235 \mathrm{~T}$ polymorphism of the AGT gene was the following: $M M-25.41 \% M T-51.64 \%$, TT $-22.95 \%$, whereas in group B: $M M-25.47 \%, M T$ $-55.28 \%, T T-19.25 \%$ and the distribution of the alleles was respectively: $M-51.23 \%, T-48.77 \%$ and $M-53.11 \%$, $T-46.89 \%$. The distributions did not differ significantly (respectively $p=0.73 ; p=0.66$ ).

Analysis of the frequency of genotypes and alleles of M235T polymorphism of the angiotensinogen gene in association with the number of endometriosis localizations is presented in Table 3. It showed no significant differences between the groups ( $p=0.19, p=0.90$ respectively for genotypes and alleles).

In the medical history of women enrolled in the study (taken from 188 women) 121 reported severe pelvic pain, whereas 67 had no such complaints. In these groups, the frequency of genotypes of the investigated AGT gene polymorphism was analyzed. In the group with pelvic pain the frequency of $M M$ genotype was $31.40 \%, M T-48.76 \%$, $T T-19.83 \%$ and in the group without pain respectively $26.87 \%, 50.75 \%, 22.39 \%$. These results did not differ statistically significantly $(p=0.79)$.

Infertility was also present in the study group $(n=127$, $44.88 \%$ ). These women underwent a laparoscopic surgical procedure to remove the foci of endometriosis. In 54 women the treatment was successful (the patients became pregnant and carried to term) and 73 women did not achieve the expected result. Both groups were compared in terms of the frequencies of $A G T$ (M235T) polymorphic genotypes. The frequencies were the following: $M M-27.78 \%, M T-44.44 \%$,

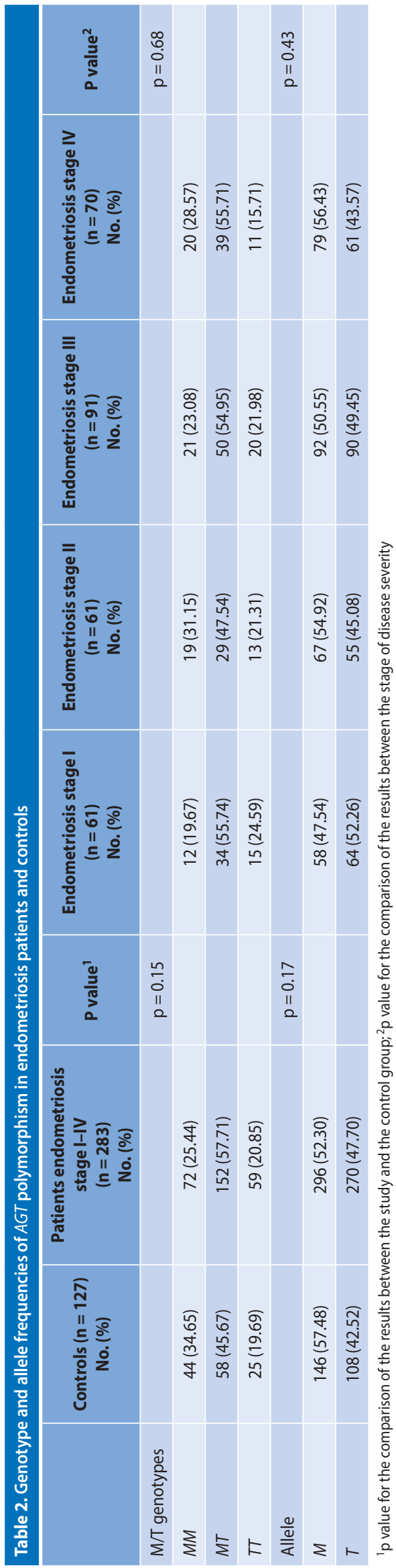


Table 3. Genotype and allele frequencies of AGT polymorphism in endometriosis patients with one, two, three, four and more endometriosis locations

\begin{tabular}{|l|c|c|c|c|c|}
\hline & $\begin{array}{c}\text { One location } \\
(\mathbf{n = 1 2 0} \\
\text { No. (\%) }\end{array}$ & $\begin{array}{c}\text { Two locations } \\
(\mathbf{n = 8 9 )} \\
\text { No. (\%) }\end{array}$ & $\begin{array}{c}\text { Three locations } \\
(\mathbf{n = 4 7 )} \\
\text { No. (\%) }\end{array}$ & $\begin{array}{c}\text { Four and more locations } \\
(\mathbf{n}=\mathbf{2 7}) \\
\text { No. (\%) }\end{array}$ & P value \\
\hline M/T genotypes & $34(28.33)$ & $20(22.47)$ & $13(27.66)$ & $5(18.52)$ & $p=0.19$ \\
\hline$M M$ & $55(45.83)$ & $57(64.04)$ & $23(48.94)$ & $17(62.96)$ & \\
\hline$M T$ & $31(25.83)$ & $12(13.48)$ & $11(23.40)$ & $5(18.52)$ & $p=0.90$ \\
\hline$T T$ & & & & & \\
\hline Allele & $124(51.24)$ & $97(54.49)$ & $49(52.13)$ & $27(50.00)$ & \\
\hline$M$ & $118(48.76)$ & $81(45.51)$ & $45(47.87)$ & $27(50.00)$ & \\
\hline$T$
\end{tabular}

TT-27.78\% and $M M-17.81 \%, M T-57.53 \%, T T-24.66 \%$, respectively for women with positive response to the treatment and no response. The observed differences were not statistically significant $(p=0.28)$.

\section{DISCUSSION}

Endometriosis is a disease characterized by the presence of an ectopic endometrium outside the uterine cavity. Endometrium is one of the few tissues in the human body that undergoes rapid cyclic growth and regression. This process involves the formation of new blood vessels. Endometrial implants require good blood supply to survive and grow. Thus, it is assumed that angiogenesis plays an important role in the pathogenesis of this disease, as confirmed by studies showing that endometrial implants are surrounded by a dense web of blood vessels [11, 12].

There are several studies on the involvement of renin-angiotensin system in the physiologic and pathologic angiogenesis. It is known that RAS-dependent formation of new blood vessels is indirect because activated angiotensin II receptors induce the expression of VEGF which is a potent pro-angiogenic factor [14]. This process appears to be tissue-specific [11].

Due to the hereditary nature of the disease, over the past several decades there has been a search for genes of specific sequence that could be associated with an increased risk of endometriosis. Among the tested genes are e.g. genes encoding proteins involved in cell cycle regulation and apoptosis, growth factors, extracellular matrix enzymes, adhesion proteins and cytokines [16]. To date, at least eight single nucleotide polymorphisms (SNPs) have been described, which seem to be related to the development of endometriosis [17].

Molecular variant M235T is one of the variants of the angiotensinogen (AGT) gene. The effect of thymine (T) replacement by cytosine $(C)$ in the $3^{\prime}$ region of exon 2 is the substitution of methionine $(M)$ for threonine $(T)$ in the encoded protein chain [18]. It appears that the $T$ allele of the analyzed polymorphism has a functional effect and is associated with increased level of angiotensinogen in plasma $[19,20]$.

In this study, the frequency of M235T polymorphism of the AGT gene was analyzed in women with endometriosis. It should be considered as pioneering research as there have been no publications in the available world literature describing the above problem. The analysis comprised 283 women in different stages of the disease according to the revised American Fertility Society (rAFS) classification [15]. There were no significant differences observed between the study group and the control group, neither between the four stages of the disease (I-IV) (Table 2) nor between the subgroups: A (stage I and II) and B (stage III and IV). Thus, the obtained results do not show the correlation between the investigated polymorphism and the development of endometriosis.

In available literature, there is no clear evidence of RAS involvement in the development and progression of endometrial foci. The most widely studied component of this system is the angiotensin I-converting enzyme ( $A C E)$ gene, which describes several polymorphisms. Hsieh et al. studied the $A C E$ insertion/deletion (I/D) gene polymorphisms in Asian women ( $n=125)$ with stage III and IV endometriosis and compared the obtained results with the control group ( $n=128)$ [21]. According to the authors, allele $/$ both in homo- (II) and heterozygous (ID) systems increased the risk of endometriosis. Different results were obtained by the authors of this paper in their previous studies [22]. The study included 241 women with endometriosis (I-IV). Apart from the comparison of the frequency of I/D polymorphism distribution between the study and the control group ( $n=127$ ), its frequency between the four stages of the disease and between stage I and II and III and IV was also analyzed. The differences were not significant in any of the analyzed systems. The study also presented the results of the determination of the frequency of angiotensin I-converting enzyme gene $A 2350 \mathrm{G}$ polymorphism and the A $1166 C$ polymorphism of the angiotensin II receptor gene 
(AT1). In the case of A1166C AT1 polymorphism, no association was found between the polymorphism and the probability of the disease development. Whereas in the case of ACE A2350G polymorphism $G$ allele and AG genotype but not $G G$, occurred significantly more frequently in the study group than in the control group $(p<0.0001)$. However, there were no differences in the frequency of individual alleles and genotypes between the different stages of the disease progression or between stage I and II and III and IV [22]. Hsieh et al. also observed that $A C E A 2350 \mathrm{G}$ gene polymorphism was associated with higher susceptibility to moderate/severe endometriosis in the Asian population [23]. In the same work the authors also analyzed another single-nucleotide polymorphism (A-240T) of the angiotensin I-converting enzyme gene located in the promoter region of the gene. In this case the $T$ allele was significantly more frequently observed in women with endometriosis compared to the control group [23]. These results were not confirmed by the study performed in Estonia [24]. The study group ( $n=150$ ) included women with all four stages of the disease. The authors failed to demonstrate associations between the A2350G and A-240T polymorphisms of the ACE gene and the increased risk of endometriosis.

Endometriosis is a disease that involves many different organs. Endometriosis foci can be localized in the peritoneum, the ovaries, fallopian tubes, parametrium, uterosacral ligaments, rectovaginal septum and also outside the reproductive organs $[6,16]$. The reason why some women develop endometriosis in one location while others in several is not known. In this study the number of organs affected by endometrial lesions varied among the examined women. Thus, four subgroups were distinguished in the study group corresponding to one, two, three and four and more locations of endometriosis. Genotype and allele frequencies of M235T polymorphism of the angiotensinogen gene were analyzed in these subgroups (Table 3). The statistical analysis did not show any significant differences between the subgroups. The results of our study may suggest that the level of angiotensinogen associated with the analyzed polymorphism (presence of $T$ allele means increased protein level) does not seem to affect the intensity of the neovascularization observed in the process of endometrial foci development.

According to the literature data endometriosis is diagnosed in 35-70\% of women with chronic pelvic pain [3, 4]. Pain is a major symptom of endometriosis but its sensation is not identical in different patients. Some of them complain of very severe pain (painful menstrual periods, PMS, pelvic pain, dyspareunia), often cyclical, which is associated with changes in sex hormone levels during the menstrual cycle. Other patients, despite significant severity of the lesions experience no pain [1]. The pain associated with endometriosis on the one hand results from the innervation of endometriotic deposits, on the other hand lesion-induced inflammatory process of nerve fibers is of significance. It appears that in the immediate vicinity and inside the endometrial implants new nerve fibers are formed, accompanied by immature blood vessels [25]. The nerve growth factor (NGF) and vascular endothelial growth factor (VEGF), the levels of which are increased in the eutopic and ectopic endometrium of women with endometriosis, are involved in both neurogenesis and angiogenesis processes $[1,6,25]$. The above data suggest a possible involvement of the neovascularization process in the perception of pain by women with endometriosis, that is why the authors of this study decided to check whether there is a correlation between the M235T polymorphism of the AGT gene and pain.

In the present study, when taking medical history concerning pelvic pain, 121 women enrolled in the study group reported pain, whereas 67 did not experience it. In this way, there were distinguished two groups that were compared as regards the frequency of the occurrence of genotypes of the investigated AGT gene polymorphism. Differences in the frequency of $M M, M T$, and TT genotypes were small and not statistically significant, suggesting that the investigated angiotensinogen gene polymorphism does not play a significant role in the etiology of pain associated with endometriosis.

Endometriosis is a disease that significantly impairs fertility. Among the women suffering from it, as many as 30 to $50 \%$ have problems with getting pregnant $[3,4]$. Often, in the absence of other ailments, it is precisely the search for the reasons for failure to become pregnant that results in the diagnosis of endometriosis. In advanced endometriosis extensive adhesions and cysts may distort pelvic anatomy which in turn contributes to infertility. In patients with minimal and moderate endometriosis anatomical changes no longer play a dominant role. Here the causes of infertility lie in inflammatory processes occurring within the peritoneal space [26].

In this research nearly half of the study group $(n=127$, $44.88 \%$ ) presented with infertility problems. These women were treated to remove the lesions and restore fertility. In some of them ( $n=54 ; 42.52 \%$ ) the therapy brought the expected effect. Both groups (with and without response to the treatment) were compared in terms of the frequency of genotypes of M235T angiotensinogen gene polymorphism. The analyzed results were differentiated but they did not show statistically significant differences. This suggests lack of the prognostic value of the investigated polymorphism as to the predicted effect of the treatment of infertility coexisting with endometriosis. 


\section{CONCLUSIONS}

No correlation was found in this study between M235T angiotensinogen gene polymorphism and the development of endometriosis, its stage or clinical course. The prognostic value of the analyzed polymorphism in the prediction of effects of the treatment of infertility coexisting with endometriosis has not been found, either.

\section{Acknowledgments}

This study was supported by the Medical University of Lodz, Poland (project no: 502-03/5-102-03/502-54-069).

\section{REFERENCES}

1. Triolo $\mathrm{O}$, Laganà $\mathrm{AS}$, Sturlese $\mathrm{E}$. Chronic pelvic pain in endometriosis: an overview. J Clin Med Res. 2013; 5(3): 153-163, doi: 10.4021/jocmr1288w, indexed in Pubmed: 23671540.

2. Mehedintu C, Plotogea MN, lonescu S, et al. Endometriosis still a challenge. J Med Life. 2014; 7(3): 349-357, indexed in Pubmed: 25408753.

3. Hompes PGA, Mijatovic V. Endometriosis: the way forward. Gynecol Endocrinol. 2007; 23(1): 5-12, indexed in Pubmed: 17484506.

4. Schliep KC, Mumford SL, Peterson CM, et al. Pain typology and incident endometriosis. Hum Reprod. 2015; 30(10): 2427-2438, doi: 10.1093/humrep/dev147, indexed in Pubmed: 26269529.

5. Sutton C. The history of endometriosis. In: Sutton C, Adamson D, Jones KD. Modern Management of Endometriosis. CRC Press, 2005 s. : 1-17.

6. Giudice LC, Kao LC. Endometriosis. Lancet. 2004; 364(9447): 1789-1799, doi: 10.1016/S0140-6736(04)17403-5, indexed in Pubmed: 15541453.

7. Ahn SH, Monsanto SP, Miller C, et al. Pathophysiology and Immune Dysfunction in Endometriosis. Biomed Res Int. 2015; 2015: 795976, doi: 10.1155/2015/795976, indexed in Pubmed: 26247027.

8. Ashrafi M, Sadatmahalleh SJ, Akhoond MR, et al. Evaluation of Risk Factors Associated with Endometriosis in Infertile Women. Int J Fertil Steril. 2016; 10(1): 11-21, indexed in Pubmed: 27123195.

9. Missmer SA, Hankinson SE, Spiegelman D, et al. Incidence of laparoscopically confirmed endometriosis by demographic, anthropometric, and lifestyle factors. Am J Epidemiol. 2004; 160(8): 784-796, doi: 10.1093/aje/kwh275, indexed in Pubmed: 15466501.

10. Hadfield RM, Mardon HJ, Barlow DH, et al. Endometriosis in monozygotic twins. Fertil Steril. 1997; 68(5): 941-942, indexed in Pubmed: 9389831.

11. Barcz E, Celińska A, Sangidori D, et al. Rola angiogenezy w powstawaniu endometriozy. Klin Perinatol Ginekol. 2006; 42(2): 12-17.

12. Taylor RN, Yu J, Torres PB, et al. Mechanistic and therapeutic implications of angiogenesis in endometriosis. Reprod Sci. 2009; 16(2): 140-146, doi: 10.1177/1933719108324893, indexed in Pubmed: 19001553.
13. Paul M, Poyan Mehr A, Kreutz R. Physiology of local renin-angiotensin systems. Physiol Rev. 2006; 86(3): 747-803, doi: 10.1152/physrev.00036.2005, indexed in Pubmed: 16816138.

14. Khakoo AY, Sidman RL, Pasqualini $R$, et al. Does the renin-angiotensin system participate in regulation of human vasculogenesis and angiogenesis? Cancer Res. 2008; 68(22): 9112-9115, doi: 10.1158/0008-5472. CAN-08-0851, indexed in Pubmed: 19010879.

15. American Fertility Society. Classification of endometriosis. Fertil Steril. 1979; 32: 633-645.

16. Rahmioglu N, Missmer SA, Montgomery GW, et al. Insights into Assessing the Genetics of Endometriosis. Curr Obstet Gynecol Rep. 2012; 1(3): 124 -137, doi: 10.1007/s13669-012-0016-5, indexed in Pubmed: 22924156.

17. Rahmioglu N, Montgomery GW, Zondervan KT. Genetics of endometriosis. Womens Health (Lond). 2015; 11(5): 577-586, doi: 10.2217/whe.15.41, indexed in Pubmed: 26441051.

18. Valdez-Velazquez LL, Quintero-Ramos A, Perez SA, et al. Genetic polymorphisms of the renin-angiotensin system in preterm delivery and premature rupture of membranes. J Renin Angiotensin Aldosterone Syst. 2007; 8(4): 160-168, doi: 10.3317/jraas.2007.026, indexed in Pubmed: 18205094.

19. Bloem LJ, Manatunga AK, Tewksbury DA, et al. The serum angiotensinogen concentration and variants of the angiotensinogen gene in white and black children. J Clin Invest. 1995; 95(3): 948-953, doi: 10.1172/JCl117803, indexed in Pubmed: 7883995.

20. Jeunemaitre $X$, Soubrier F, Kotelevtsev YV, et al. Molecular basis of human hypertension: role of angiotensinogen. Cell. 1992; 71(1): 169-180, indexed in Pubmed: 1394429.

21. Hsieh YY, Lee CC, Chang CC, et al. Angiotensin I-converting enzyme insertion-related genotypes and allele are associated with higher susceptibility of endometriosis and leiomyoma. Mol Reprod Dev. 2007; 74(7): 808-814, doi: 10.1002/mrd.20474, indexed in Pubmed: 17186537.

22. Kowalczyńska LJ, Ferenc T, Wojciechowski M, et al. Endometriosis and RAS system gene polymorphisms: the association of ACE A2350G polymorphism with endometriosis in Polish individuals. DNA Cell Biol. 2014; 33(5): 328-335, doi: 10.1089/dna.2013.2255, indexed in Pubmed: 24571615.

23. Hsieh YY, Chang CC, Tsai FJ, et al. Angiotensin I-converting enzyme ACE $2350^{*} \mathrm{G}$ and ACE-240*T-related genotypes and alleles are associated with higher susceptibility to endometriosis. Mol Hum Reprod. 2005; 11(1): 11-14, doi: 10.1093/molehr/gah131, indexed in Pubmed: 15531592.

24. Lamp M, Saare M, Laisk T, et al. Genetic variations in vascular endothelial growth factor but not in angiotensin I-converting enzyme genes are associated with endometriosis in Estonian women. Eur J Obstet Gynecol Reprod Biol. 2010; 153(1): 85-89, doi: 10.1016/j.ejogrb.2010.07.021, indexed in Pubmed: 20685027.

25. Morotti M, Vincent $K$, Brawn J, et al. Peripheral changes in endometriosis-associated pain. Hum Reprod Update. 2014; 20(5): 717-736, doi: 10.1093/humupd/dmu021, indexed in Pubmed: 24859987.

26. Gupta S, Goldberg JM, Aziz N, et al. Pathogenic mechanisms in endometriosis-associated infertility. Fertil Steril. 2008; 90(2): 247-257, doi: 10.1016/j.fertnstert.2008.02.093, indexed in Pubmed: 18672121. 